\title{
Psychological processing of a kidney transplantation, perceived quality of life, and immunosuppressant medication adherence
}

This article was published in the following Dove Press journal:

Patient Preference and Adherence

\author{
Jennifer Scheel' \\ Katharina Schieber' \\ Sandra Reber' \\ Sabine Jank ${ }^{2}$ \\ Kai-Uwe Eckardt ${ }^{2}$ \\ Franziska Grundmann ${ }^{3}$ \\ Frank Vitinius ${ }^{4}$ \\ Martina de Zwaan ${ }^{5}$ \\ Anna Bertram 6 \\ Yesim Erim' \\ 'Department of Psychosomatic Medicine \\ and Psychotherapy, University Hospital \\ Erlangen, Friedrich-Alexander University \\ Erlangen-Nürnberg (FAU), Erlangen, \\ Germany; ${ }^{2}$ Department of Nephrology and \\ Hypertension, University Hospital Erlangen, \\ Friedrich-Alexander University Erlangen- \\ Nürnberg (FAU), Erlangen, Germany; \\ ${ }^{3}$ Department II of Internal Medicine, \\ Nephrology, Rheumatology, Diabetes and \\ General Internal Medicine, University \\ Hospital of Cologne, Cologne, Germany; \\ ${ }^{4}$ Department of Psychosomatics and \\ Psychotherapy, University Hospital of \\ Cologne, Cologne, Germany; ${ }^{5}$ Department \\ of Psychosomatic Medicine and \\ Psychotherapy, Hannover Medical School, \\ Germany; ${ }^{6}$ Department of Nephrology and \\ Hypertension, Hannover Medical School, \\ Hanover, Germany
}

Introduction: Though psychosocial well-being and quality of life generally improve after transplantation, a relevant proportion of patients suffers from psychosocial problems. Further analysis of the psychological coping after kidney transplantation is needed to identify patients at risk. The aim of this study was to examine the psychological response after kidney transplantation and its associations with health-related quality of life and immunosuppressant medication adherence.

Materials and methods: The coping process after kidney transplantation was investigated with the Transplant Effects Questionnaire (TxEQ; subscales: worry, guilt, disclosure, adherence, responsibility) in 267 adult kidney transplant recipients $\geq 12$ months post-transplantation. Furthermore, perceived health-related quality of life, self-reported immunosuppressant medication adherence, and sub-therapeutic immunosuppressant trough levels as biological markers of adherence were assessed.

Results: Patients showed moderate scores concerning the subscales "worry", "guilt", and "responsibility" as well as high scores concerning "disclosure". Except for "adherence", all TxEQ subscales were associated with mental, but not with physical health-related quality of life and self-reported adherence. Sub-therapeutic immunosuppressant trough levels were significantly associated only with the TxEQ subscale "worry".

Conclusions: The present results suggest a conditional structure in which mental health-related quality of life is negatively associated with worries, guilt, and responsibility and positively with disclosure. Adherence seems to be a complex behavior, which is not necessarily directly associated with the psychological processing of organ transplantations. As mental healthrelated quality of life is related to this psychological processing, the TxEQ could be used as a screening tool for problematic psychological processing after kidney transplantation.

Keywords: kidney transplantation, psychological processing, Transplant Effects Questionnaire, health-related quality of life, adherence, immunosuppressant trough levels

\section{Introduction}

Psychosocial well-being and quality of life generally improve after organ transplantation (heart, kidney, liver, and lung). ${ }^{1-5}$ Transplant patients' quality of life is mainly reported as nearly equivalent to the general population, ${ }^{5}$ although, a relevant proportion of patients suffers from psychological or psychosocial problems. ${ }^{1,2}$ A transplantation is an extraordinary bodily experience with particular requirements regarding emotional coping and illness acceptance. Besides the physical adjustment, also a psychological adaptation to the transplant is necessary. ${ }^{6}$
Correspondence: Yesim Erim Department of Psychosomatic Medicine and Psychotherapy, University Hospital Erlangen, Friedrich-Alexander University ErlangenNürnberg (FAU), Schwabachanlage 6, 91054 Erlangen, Germany

Email yesim.erim@uk-erlangen.de 
Kidney transplantation is the most frequent solid organ transplantation in Germany. ${ }^{7}$ Yet besides the medical advantages of transplantation, it causes new stressors, eg, the challenge of adherence to immunosuppressant medication and the maintenance of a healthy lifestyle as well as coping with emotional problems like worry, anxiety, or feelings of guilt. ${ }^{5,8}$ Renal transplant patients worry significantly more about their transplant and experience significantly more feelings of guilt after transplantation than recipients of other solid organs like heart and lung. ${ }^{6}$ The identification of patients at risk, who experience high mental distress, is of high clinical importance, as low mental health-related quality of life is related to nonadherence. ${ }^{9}$ The high psychosocial distress of these patients is also reflected by the fact that about $40 \%$ of kidney transplant recipients report a current interest in a support group. ${ }^{10}$

Because of the need to assess the integration of a new organ, various instruments have been released. The Transplant Effects Questionnaire (TxEQ) has been developed to assess the emotional reaction to organ transplantations as well as the health behavior in the aftermath (TxEQ; Ziegelmann et al, ${ }^{5}$ German translation and validation by Klaghofer et $\mathrm{al}^{11}$ ). With the TxEQ, the cognitive, emotional and behavioral responses after organ transplantations are assessed in the dimensions "worry", "guilt", "disclosure", "responsibility", and "adherence". ${ }^{11}$

Furthermore, the psychological processing after kidney transplantation is associated with health-related quality of life: Klaghofer et a ${ }^{11}$ could demonstrate significant correlations of all TxEQ subscales and the mental component scale of SF-36 $(0.17 \leq r \leq 0.39)$, but only of the subscales "worry" and "disclosure" with the physical component scale. Similarly, Kim et $\mathrm{al}^{12}$ and Griva et $\mathrm{al}^{8}$ reported TxEQ subscales to be associated with mental quality of life, but not with physical health-related quality of life. Both found "worry" to be significantly associated with poorer mental health-related quality of life. ${ }^{8,12}$ Furthermore, Kim et al ${ }^{12}$ reported "guilt" to be significantly associated with poorer mental health-related quality of life.

Especially in the light of the ongoing debate regarding reproducibility of scientific findings (eg, Heino et al), replication is required. More evidence concerning the association of coping with organ transplantations and health outcomes is needed. Therefore, the aim of this study was to investigate the associations of psychological processing after organ transplantation with perceived quality of life, self-reported immunosuppressant adherence, and sub-therapeutic immunosuppressant trough levels, which are risk factors for graft rejection, ${ }^{13}$ but have not yet been investigated in the present context.

We hypothesized a similar TxEQ pattern after kidney transplantation as presented by Goetzmann et al, ${ }^{6}$ Griva et $\mathrm{al}^{8}{ }^{8}$ and Kim et al. ${ }^{12}$ Furthermore, we hypothesized nonadaptive psychological processing to be associated with low health-related quality of life, immunosuppressant medication nonadherence, and sub-therapeutic immunosuppressant trough levels. In detail, we expected associations of the TxEQ subscales with mental but not with physical health-related quality of life, as indicated by the studies mentioned above. ${ }^{6,8,12}$

\section{Materials and methods}

\section{Patients}

This multicenter study was performed at the transplant centers of the University Hospitals of Erlangen and Cologne and of Hannover Medical School and included 267 consecutive adult patients, who had undergone kidney transplantation between 1981 and 2014 and who presented at the transplant center for follow-up examinations between November 2014 and December 2015. The present study was conducted within a large multicenter project with disparate research questions and many different dependent and independent variables, which make it impossible to publish the whole project in one article. Therefore, the data set has in parts already been published with distinct foci.

Exclusion criteria encompassed being $<1$ year posttransplantation, additional non-kidney transplantation, availability of $<4$ immunosuppressant trough levels within the last 12 months before the assessment (levels within the first 6 months after transplantation were not included; thus, in patients who received the organ 12 months before study assessment, trough levels of only 6 months were included), mental disability, and insufficient German language skills for understanding of the questionnaires.

Institutional ethics committee approval was obtained in each participating institution, and all participants gave written informed consent. Trained medical staff, which was not part of the transplant team, approached the patients and distributed the questionnaires. Immunosuppressant trough levels from the last 6-12 months before the assessment were recorded from the patient charts.

\section{Transplant Effects Questionnaire}

The Transplant Effects Questionnaire (TxEQ; ${ }^{5}$ ) was used in the German version. ${ }^{11}$ It assesses the emotional response to 
receiving a transplant as well as the health behavior of a transplant recipient. The questionnaire consists of the five subscales "worry" ( 6 items, range $=6-30$, higher scores indicate more worry), "guilt" ( 5 items, range $=5-25$, higher scores indicate more feelings of guilt), "disclosure" ( 3 items, range $=3-15$, higher scores indicate more disclosure), "responsibility" ( 4 items, range $=4-20$, higher scores indicate higher responsibility), and "adherence" (5 items, range $=5-25$, higher scores indicate better adherence behavior). The items are rated on a 5-point scale from 1 ("strongly agree") to 5 ("strongly disagree"). The subscales are calculated by (1) recoding items 1, 3, 4, 6, 9, 10, 12, 14, 16, 17, 18, 19,21 , and 23 , (2) summing the respective items for each subscale, and (3) dividing these sums by the respective number of items. Goetzmann et $\mathrm{al}^{6}$ suggested the following cutoff scores: $>3.5$ for high worry and high guilt; $<2.5$ for low disclosure, responsibility, and adherence.

With regard to internal consistency, we found Cronbach's alpha to be 0.75 for the whole TxEQ (23 items), and $0.71-0.78$ for the subscales (comparable to 0.71-0.79 found by Klaghofer et $\mathrm{al}^{11}$ for the German version and to $0.72-0.86$ found by Ziegelmann et $\mathrm{al}^{5}$ for the English original version).

\section{Perceived health-related quality of life}

Perceived health-related quality of life was measured by the short version of the Short Form Health Survey SF-36 (SF$12 ;{ }^{14}$ German version by Bullinger et $\mathrm{al}^{15}$ and validation by Gandek et al; ${ }^{16}$ self-report instrument). The SF-12 consists of the two subscales "Mental Component Summary" (MCS) and "Physical Component Summary" (PCS), which both range from 0 to 100 . Higher scores indicate higher subjectively perceived health-related quality of life.

\section{Self-reported immunosuppressant medication adherence}

The Basel Assessment of Adherence to Immunosuppressant Medications Scale (BAASISC ${ }^{17}$ German version by the Leuven-Basel Adherence Research Group, ${ }^{18}$ self-report instrument) was used to assess self-reported immunosuppressant medication adherence. It comprises four items concerning adherence to immunosuppressant medication (dose taking, drug holidays, timing deviation $>2$ hrs from prescribed time, dose reduction) on a 6 -point scale $(0=$ never, $5=$ every day). Nonadherence is defined as at least one affirmative answer to any of the four items (dichotomous score). Additionally, we calculated a continuous adherence score by summing the scores of the four single items (range: $0-20$ ), as suggested by De Bleser et $\mathrm{al}^{19}$ and Pabst et $\mathrm{al}^{20}$. Following a systematic review concerning self-reported medication nonadherence, the BAASISC is recommended as a reliable, valid, and sensitive tool. ${ }^{17}$

\section{Sub-therapeutic immunosuppressant trough levels}

Sub-therapeutic immunosuppressant trough levels (tacrolimus, cyclosporine, everolimus, and sirolimus) were employed as a biological marker of adherence. Immunosuppressant trough levels were assessed from EDTA blood samples via LC-MS/MS (liquid chromatography mass spectrometry/mass spectrometry) in Erlangen and Hannover and via immune-assays in Cologne. IS trough levels were excluded as invalid, if they were measured during hospitalization or apparently shortly after IS intake. The minimal number of valid immunosuppressant trough levels for the calculation of the percentage of subtherapeutic trough levels was 4 , as medication levels from various time points are assumed to be more valid than a single medication level. ${ }^{20}$ The percentage of subtherapeutic immunosuppressant trough levels was calculated by dichotomizing every single immunosuppressant trough level for each patient in $<$ or $\geq$ the respective target level (immunosuppressant target levels are determined individually for each patient and can be changed by the transplant physicians due to the clinical course, therefore, they can differ both intra-patient and inter-patient) and counting the number of sub-therapeutic immunosuppressant trough levels for each patient $(0-100 \%)$.

\section{Statistical analyses}

In the case of missing variables, no imputation was conducted, but the patient was excluded from the respective analyses. As 2 of the 267 patients did not provide self-reported adherence data, they were excluded from the analyses regarding these variables. For an overview, means, SDs, minima and maxima, and frequencies (as appropriate) were given of all variables. For the description of simple relationships, Pearson correlation coefficients were computed. Group differences were analyzed using Mann-Whitney $U$ tests for not normally distributed continuous variables (effect size measure: $r ; r \geq 0.1$ small, $r \geq 0.3$ medium, $r \geq 0.5$ large effect size ${ }^{21}$ ). For all analyses, the statistical analysis program SPSS 21 (IBM; Armonk, NY, USA) was used. Findings were considered to be statistically significant at $\alpha<0.05$. 


\section{Results}

\section{Description of the sample}

A total of 267 patients were included in the analyses. 34.8\% of the patients were female ( $\mathrm{n}=93$ ); the mean current age was $52.8 \pm 13.7$ years and the mean time since the last transplantation was $7.1 \pm 6.2$ years.

\section{Psychological processing of a kidney transplantation}

The mean TxEQ scores of the total sample ranged from 1.9 to 4.4. The mean scores of the whole sample concerning "worry" and "guilt" were below the cutoff of 3.5 suggested by Goetzmann et $\mathrm{al}^{6}$ for high worry and high guilt. The mean scores of the whole sample concerning "responsibility", "adherence", and "disclosure" were above the cutoff of 2.5 suggested by Goetzmann et $\mathrm{al}^{6}$ for low disclosure, responsibility, and adherence. For details, see Table 1. When applying the cutoff scores according to Goetzmann et al, ${ }^{6} 16.1 \%(\mathrm{n}=43)$ experienced high worry $(>3.5), 1.5 \%(\mathrm{n}=4)$ high guilt $(>3.5), 2.6 \%$ $(\mathrm{n}=7)$ appraised disclosure as problematic $(<2.5), 1.1 \%$ $(n=3)$ exhibited low adherence scores $(<2.5)$, and $34.8 \%$ $(n=93)$ felt low responsibility for their transplant $(<2.5)$. Regarding "responsibility", $21.3 \%$ of the patients $(n=57)$ scored in the lowest quartile of possible "responsibility" scores $(<2.0), 69.0 \%$ of the patients $(n=184)$ scored in the two middle quartiles of possible "responsibility" scores $(2.0 \leq x \leq 4.0)$, and $9.7 \%$ of the patients $(n=26)$ scored in the highest quartile of possible "responsibility" scores $(>4.0)$; the median was 3.0.

The five TxEQ scales were significantly correlated with each other (except for "adherence" with both "worry" and "responsibility"; for details see Table 2). Male $(\mathrm{n}=174 ; 65 \%)$ and female $(\mathrm{n}=93 ; 35 \%)$ patients did not differ significantly concerning any TxEQ subscales (Mann-Whitney tests, Table 1).

\section{Perceived health-related quality of life and psychological processing after kidney transplantation}

Mental health-related quality of life was significantly negatively correlated with the TxEQ subscales "worry", "guilt", and "responsibility" and significantly positively correlated with "disclosure", but not with "adherence". Physical health-related quality of life was not correlated with any of the TxEQ subscales. For detailed descriptive data regarding perceived healthrelated quality of life see Table 3 .

Table I Comparison of TxEQ subscale scores by sex

\begin{tabular}{|l|l|l|l|l|l|}
\hline TxEQ subscales & & Females (n=93) & Males (n= I 74) & $\boldsymbol{p}$ (Mann-Whitney U test) & Effect size (r) \\
\hline Worry & M \pm SD (range) & $2.9 \pm 0.8(1.3-4.7)$ & $2.8 \pm 0.8(01.0-4.7)$ & Not significant & 0.1 \\
Guilt & $M \pm$ SD (range) & $2.0 \pm 0.6(1.0-3.4)$ & $1.9 \pm 0.7(1.0-4.0)$ & Not significant & 0.1 \\
Disclosure & $M \pm$ SD (range) & $4.4 \pm 0.7(2.3-5.0)$ & $4.4 \pm 0.7(1.7-5.0)$ & Not significant & 0.0 \\
Responsibility & $M \pm$ SD (range) & $2.7 \pm 1.0(1.0-4.5)$ & $2.9 \pm 1.1(1.0-5.0)$ & Not significant & -0.1 \\
Adherence & $M \pm$ SD (range) & $4.4 \pm 0.6(2.0-5.0)$ & $4.4 \pm 0.6(1.6-5.0)$ & Not significant & 0.0 \\
\hline
\end{tabular}

Abbreviations: M, mean; SD, Standard Deviation; TxEQ, Transplant Effects Questionnaire.

Table 2 Descriptive data of the TxEQ subscales and their correlative associations

\begin{tabular}{|c|c|c|c|c|c|c|c|}
\hline & & & \multicolumn{5}{|c|}{ TxEQ subscales } \\
\hline & & & Worry & Guilt & Disclosure & Responsibility & Adherence \\
\hline \multicolumn{8}{|c|}{ TxEQ subscales } \\
\hline Worry & $M \pm S D$ (range) & $2.8 \pm 0.8(1.0-4.7)$ & & $0.4^{* *}$ & $-0.3^{* *}$ & $0.5 * *$ & -0.1 \\
\hline Guilt & $M \pm S D$ (range) & $1.9 \pm 0.7(1.0-4.0)$ & & & $-0.3 * *$ & $0.5 * *$ & $-0.2 * *$ \\
\hline Disclosure & $M \pm S D$ (range) & $4.4 \pm 0.7(1.7-5.0)$ & & & & $-0.2^{* *}$ & $0.3^{* *}$ \\
\hline Responsibility & $M \pm S D$ (range) & $2.8 \pm 1.0(1.0-5.0)$ & & & & & -0.1 \\
\hline Adherence & $M \pm S D$ (range) & $4.4 \pm 0.6(1.6-5.0)$ & & & & & \\
\hline
\end{tabular}

Notes: ${ }^{* *} p \leq 0.001 ;{ }^{*} p<0.05$.

Abbreviations: M, mean; SD, Standard Deviation; TxEQ, Transplant Effects Questionnaire. 
Table 3 Descriptive data of perceived health-related quality of life, self-reported immunosuppressant medication nonadherence, and sub-therapeutic immunosuppressant trough levels and their correlative associations with the TxEQ subscales

\begin{tabular}{|c|c|c|c|c|c|c|c|}
\hline & & & \multicolumn{5}{|c|}{ TxEQ subscales } \\
\hline & & & Worry & Guilt & Disclosure & Responsibility & Adherence \\
\hline \multicolumn{8}{|c|}{ Immunosuppressant medication variables } \\
\hline Nonadherence (BAASISC), dichotomous & Yes: $\mathrm{n}, \%$ & $87(33.3 \%)$ & 0.0 & 0.0 & -0.0 & -0.1 & $-0.4^{* *}$ \\
\hline Nonadherence (BAASIS@), continuous & $\mathrm{M} \pm \mathrm{SD}$ (range) & $0.6 \pm 1.1(0.0-6.0)$ & 0.0 & 0.0 & -0.1 & -0.1 & $-0.4 * *$ \\
\hline $\begin{array}{l}\text { Percentage of sub-therapeutic immuno- } \\
\text { suppressant trough levels }\end{array}$ & $M \pm S D$ (range) & $40 \pm 30 \%(0-100)$ & $0.1^{*}$ & 0.1 & -0.1 & 0.1 & 0.0 \\
\hline \multicolumn{8}{|l|}{ Perceived health-related quality of life } \\
\hline $\begin{array}{l}\text { Physical health-related quality of life } \\
\text { (SF-12, PCS) }\end{array}$ & $M \pm S D$ (range) & $\begin{array}{l}44.9 \pm 10.6 \\
(15.7-67.7)\end{array}$ & -0.1 & -0.1 & 0.1 & -0.0 & -0.0 \\
\hline $\begin{array}{l}\text { Mental health-related quality of life (SFI2, } \\
\text { MCS) }\end{array}$ & $M \pm S D$ (range) & $\begin{array}{l}49.3 \pm 10.2 \\
(19.6-66.5)\end{array}$ & $-0.3^{* *}$ & $-0.3^{* *}$ & $0.3^{* *}$ & $-0.1^{*}$ & 0.1 \\
\hline
\end{tabular}

Notes: ${ }^{* *} p \leq 0.001 ; *_{p}<0.05$

Abbreviations: M, mean; SD, Standard Deviation; TxEQ, Transplant Effects Questionnaire.

\section{Self-reported immunosuppressant medication adherence and psychological processing after kidney transplantation}

$33.3 \%$ of the patients admitted to be nonadherent in the BAASISC $C$. Adherence according to the BAASISC was significantly positively correlated with adherence according to the TxEQ (though only $1.1 \%$ of the patients exhibited adherence scores below the cutoff of 2.5 in this instrument). Regarding the other TxEQ subscales, there were no significant associations with the two adherence measures (see Table 3).

\section{Sub-therapeutic immunosuppressant trough levels and psychological processing after kidney transplantation}

The percentages of sub-therapeutic immunosuppressant trough levels were significantly correlated only with the TxEQ subscale "worry". Sub-therapeutic immunosuppressant trough levels were not correlated with self-reported immunosuppressant medication adherence, neither the BAASIS $\odot$ nor the TxEQ subscale "adherence" (see Table 3).

\section{Discussion}

Our main findings are as follows: The TxEQ subscales "worry", "guilt", "disclosure", and "responsibility" were related to mental health-related quality of life, but not to physical health-related quality of life. Adherence measured by the BAASISC was positively associated with adherence measured by the TxEQ, but not to any other TxEQ subscale.
Adherence does not appear to be associated with a specific way of processing organ transplantation.

\section{Associations of the TxEQ subscales and health-related quality of life}

As to be expected from the studies by Goetzmann et al, ${ }^{6}$ Griva et $\mathrm{al}^{8}{ }^{8}$ and Kim et al, ${ }^{12}$ we found associations of the TxEQ subscales with mental, but not with physical health-related quality of life. Kim et $\mathrm{al}^{12}$ and Griva et $\mathrm{al}^{8}$ both reported "worry" to be negatively associated with mental healthrelated quality of life and Kim et al ${ }^{12}$ additionally reported "guilt" to be negatively associated with mental health-related quality of life. We could confirm these associations in our sample and additionally found a negative association with "responsibility" and a positive association with "disclosure".

The TxEQ subscales are related to the experience of transplantation. On the one hand, it is comprehensible that there may be concerns about losing the organ, which possibly leads to a responsible handling of medication and medical aftercare. On the other hand, too many worries may lead to depressed mood and therefore low mental health-related quality of life. Also feelings of guilt regarding the deceased or living donor could lead to depressed mood and induce a very high feeling of personal responsibility and maintain responsible behavior. Yet, a very high feeling of personal responsibility to manage everything related to the transplantation perfectly might, in turn, lead to distress, depressive symptoms, and even more feelings of guilt. The strongest intercorrelations of TxEQ subscales are those between "responsibility" and both "guilt" and "worry". The present 
results suggest a conditional structure of limited mental health-related quality of life and the triad worries, guilt, and responsibility. That the willingness to disclose the transplant experience in social contacts is linked to higher mental quality of life is conceivable, as openness requires a stable and positive mood and a certain self-confidence, which are often related to high quality of life.

\section{Assessment of immunosuppressant medication adherence}

The significant, but only moderate correlation of the two self-report measures of adherence (TxEQ subscale "adherence" and BAASIS(C) again highlights the difficulties concerning convergent validity in the assessment of medication adherence. Different assessment tools seem to measure different aspects of adherence. Furthermore, selfreported immunosuppressant medication nonadherence was not associated with sub-therapeutic immunosuppressant trough levels (neither via BAASIS $\odot$, nor via TxEQ). In future studies on this topic, electronic medication monitoring systems could be added, as they are a more objective reference method for adherence.

\section{Association of worrying and percentage of sub-therapeutic trough levels}

The TxEQ subscale "worry" was positively correlated with the percentage of sub-therapeutic immunosuppressant trough levels. Yet, both worrying and the percentage of sub-therapeutic immunosuppressant trough levels were not associated with self-reported adherence behavior (neither via BAASIS $\odot$ nor via TxEQ). Therefore, high worrying might be a consequence of subtherapeutic immunosuppressant trough levels, which seem to be uncontrollable despite good self-perceived adherence behavior. ${ }^{13}$

\section{Limitations}

There are some limitations to our study. First, the retrospective, cross-sectional design does not allow for causal conclusions regarding the associations between TxEQ and other variables. Second, a possible recruiting bias (eg, nonadherence and problems in disclosing oneself as a transplant recipient might be related to nonparticipation) and a possible influence of social desirability on answers in self-report measures must be considered in patients contacted during their scheduled visits to the transplantation center.

\section{Conclusion}

The psychological processing of organ transplantation is associated with mental health-related quality of life. The present results suggest a conditional structure in which limited mental health-related quality of life is associated with worries, guilt and responsibility, whereas high disclosure is associated with higher mental quality of life. Against our expectations, we could not find associations between the emotional aspects of coping after transplantation and the selfreported immunosuppressant medication adherence. Also, the percentage of sub-therapeutic immunosuppressant trough levels as a biological marker of adherence was associated only with worrying. Adherence seems to be a complex behavior, which is not necessarily directly associated with parameters of psychological processing of organ transplantations like worries, guilt or responsibility. The TxEQ gives a profile of the mechanisms used for coping with transplantation as a stressful life event. Furthermore, the TxEQ can be used as a screening tool for the identification of patients with problematic psychological processing after kidney transplantation, especially regarding strong worrying in the course of the psychological processing after kidney transplantation, which was experienced by $16.1 \%$ of the patients.

\section{Ethics approval and consent to participate}

Institutional ethics committee approval was obtained in each participating institution [Ethics Committee of the University Hospital Erlangen, Friedrich-Alexander University ErlangenNürnberg (FAU), Ethics Committee of the Hannover Medical School, Ethics Committee of the University Hospital of Cologne] and has been performed in accordance with the ethical standards as laid down in the 1964 Declaration of Helsinki and its later amendments or comparable ethical standards. All participants gave written informed consent.

\section{Data availability}

The authors have full control of all primary data and agree to allow the journal to review their data if requested.

\section{Acknowledgments}

We thank Prof. Lutz Götzmann for his valuable advice and inspiration. We thank Lisa Stößel for her support in obtaining the data. This research did not receive any specific grant from funding agencies in the public, commercial, or not-for-profit sectors. 


\section{Author contributions}

All authors contributed to data analysis, drafting or revising the article, gave final approval of the version to be published, and agree to be accountable to all aspects of the work. Present address for JS is Center for Health Services Research in Medicine, Department of Psychiatry and Psychotherapy, University Hospital Erlangen, Friedrich-AlexanderUniversity Erlangen-Nürnberg (FAU), Germany; Present address for SJ is Medical Practice for Internal Medicine and Nephrology of MD Stefan Graf \& MD Sabine Jank; Nürnberg, Germany; Present address for KUE is Department of Nephrology and Medical Intensive Care, Charité - Universitätsmedizin Berlin, Berlin, Germany.

\section{Disclosure}

Prof. Dr. Martina de Zwaan report personal fees from NovoNordisk, outside the submitted work. The authors report no other conflicts of interest in this work.

\section{References}

1. Goetzmann L, Ruegg L, Stamm M, et al. Psychosocial profiles after transplantation: a 24-month follow-up of heart, lung, liver, kidney and allogeneic bone-marrow patients. Transplantation. 2008;86 (5):662-668. doi:10.1097/TP.0b013e3181817dd7

2. Engle D. Psychosocial aspects of the organ transplant experience: what has been established and what we need for the future. J Clin Psychol. 2001;57(4):521-549. doi:10.1002/(ISSN)1097-4679

3. Dew MA, Switzer GE, Goycoolea JM, et al. Does transplantation produce quality of life benefits? A quantitative analysis of the literature. Transplantation. 1997;64(9):1261-1273. doi:10.1097/00007890199711150-00006

4. Goetzmann L, Scholz U, Dux R, et al. Attitudes towards transplantation and medication among 121 heart, lung, liver and kidney recipients and their spouses. Swiss Med Wkly. 2012;142:w13595.

5. Ziegelmann JP, Griva K, Hankins M, et al. The Transplant Effects Questionnaire (TxEQ): the development of a questionnaire for assessing the multidimensional outcome of organ transplantation - example of End Stage Renal Disease (ESRD). Br J Health Psychol. 2002;7(Part 4):393-408. doi:10.1348/135910702320645381

6. Goetzmann L, Sarac N, Ambuhl P, et al. Psychological response and quality of life after transplantation: a comparison between heart, lung, liver and kidney recipients. Swiss Med Wkly. 2008;138(33-34):477-483.

7. dso.de [Homepage on the Internet]. Statistics regarding organ transplantation in Germany. [updated October 10, 2018; cited November 5, 2018]. Available from: https://www.dso.de/. Accessed November 5, 2018.
8. Griva K, Ziegelmann JP, Thompson D, et al. Quality of life and emotional responses in cadaver and living related renal transplant recipients. Nephrol Dial Transplant. 2002;17(12):2204-2211.

9. Scheel JF, Schieber K, Reber S, et al. Psychosocial variables associated with immunosuppressive medication non-adherence after renal transplantation. Front Psychiatry. 2018;9:23. doi:10.3389/fpsyt.2018.00023

10. Brijmohan A, Famure O, Sihota K, Shea M, Marzario B, Mitchell M. Psychosocial needs assessment post kidney transplant: feasibility of a post-transplant specific support group. CANNT J. 2015;25(3):14-21.

11. Klaghofer R, Sarac N, Schwegler K, et al. [Questionnaire on emotional response after organ transplantation: German validation of the Transplant Effect Questionnaire (TxEQ-D)]. Z Psychosom Med Psychother. 2008;54(2):174-188.

12. Kim I-K, Bae SH, Son S, Kim MS, Jun SY, Ju MK. Health-related quality of life and psychologic distress in Korean kidney transplant recipients. Transplant Proc. 2016;48(3):855-857. doi:10.1016/j. transproceed.2015.12.084

13. Scheel J, Reber S, Stoessel L, et al. Patient-reported non-adherence and immunosuppressant trough levels are associated with rejection after renal transplantation. BMC Nephrol. 2017;18(1):107. doi:10.1186/s12882-017-0669-4

14. Ware JE Jr, Kosinski M, Keller S. A 12-item shortform health survey: construction of scales and preliminary tests of reliability and validity. Med Care. 1996;34:220-233. doi:10.1097/00005650-19960300000003

15. Bullinger M, Kirchberger I. Sf-36 Fragebogen Zum Gesundheitszustand. Göttingen: Hogrefe-Verlag; 1998.

16. Gandek B, Ware JE, Aaronson NK, et al. Cross-validation of item selection and scoring for the Sf-12 health survey in nine countries: results from the IQOLA Project. International Quality of Life Assessment. J Clin Epidemiol. 1998;51(11):1171-1178. doi:10.1016/S0895-4356(98)00109-7

17. Dobbels F, Berben L, De Geest S, et al. The psychometric properties and practicability of self-report instruments to identify medication nonadherence in adult transplant patients: a systematic review. Transplantation. 2010;90(2):205-219. doi:10.1097/TP.0b013 e3181da1933

18. Leuven-Basel Adherence Research Group, Institute of Nursing Science, University of Basel, Basel, Switzerland. Basel Assessment of Adherence to Immunosuppressant Medications Scale (Baasis@). 2005.

19. De Bleser L, Dobbels F, Berben L, et al. The spectrum of nonadherence with medication in heart, liver, and lung transplant patients assessed in various ways. Transpl Int. 2011;24(9):882-891. doi:10.1111/j.1432-2277.2011.01296.x

20. Pabst S, Bertram A, Zimmermann T, Schiffer M, de Zwaan M. Physician reported adherence to immunosuppressants in renal transplant patients: prevalence, agreement, and correlates. J Psychosom Res. 2015;79(5):364-371. doi:10.1016/j.jpsychores.2015.09.001

21. Cohen J. Statistical Power Analysis for the Behavioural Sciences. 2nd ed. New York: Academic Press; 1988. 


\section{Publish your work in this journal}

Patient Preference and Adherence is an international, peer-reviewed, open access journal that focusing on the growing importance of patient preference and adherence throughout the therapeutic continuum. Patient satisfaction, acceptability, quality of life, compliance, persistence and their role in developing new therapeutic modalities and compounds to optimize clinical outcomes for existing disease

states are major areas of interest for the journal. This journal has been accepted for indexing on PubMed Central. The manuscript management system is completely online and includes a very quick and fair peer-review system, which is all easy to use. Visit http:// www.dovepress.com/testimonials.php to read real quotes from published authors.

Submit your manuscript here: https://www.dovepress.com/patient-preference-and-adherence-journal 